\title{
Chronic bilateral dislocation of temporomandibular joint
}

\author{
Shakya $\mathrm{S}^{1}$, Ongole $\mathrm{R}^{2}$, Sumanth $\mathrm{KN}^{2}$, Denny $\mathrm{CE}^{3}$
}

${ }^{1}$ Specialist Resident, ${ }^{2}$ Associate Professor, ${ }^{3}$ Assistant Professor, Department of Oral Medicine and Radiology, Manipal College of Dental Sciences, Mangalore, India

\begin{abstract}
Dislocation of the condyle of the mandible is a common condition that may occur in an acute or chronic form. It is characterised by inability to close the mouth with or without pain. Dislocation has to be differentiated from subluxation which is a self reducible condition.

Dislocation can occur in any direction with anterior dislocation being the commonest one. Various predisposing factors have been associated with dislocation like muscle fatigue and spasm, the defect in the bony surface like shallow articular eminence, and laxity of the capsular ligament. People with defect in collagen synthesis like Ehler Danlos syndrome, Marfan syndrome are said to be genetically predisposed to this condition.

Various treatment modalities have been used ranging from conservative techniques to surgical methods. Acute dislocations can be reduced manually or with conservative approach and recurrent and chronic cases can be reduced by surgical intervention. Though the dislocation in our case was 4 months a simple manual reduction proved to be successful. We believe that manual reduction can be attempted as first line of treatment prior to surgical intervention.
\end{abstract}

Key words: Temporomandibular joint, Dislocation, Management

$\mathrm{D}$ islocation of Temporomandibular joint (TMJ), also known as "Open lock" is the separation of the articular surfaces ${ }^{1}$. The condition is characterised by inability to close the mouth after wide opening. Normally, in maximum opening position the condyle is in relation to the posterior slope of the articular eminence. In case of dislocation, the condyle is not in its regular position and may be placed anterior, posterior, superior, medial or lateral to the glenoid fossa. The most common type is the anterior dislocation ${ }^{2}$. Bilateral dislocation is more common than unilateral dislocation. However, when the dislocation is unilateral, the chin is deviated to the contralateral side. Palpation in the preauricular region reveals an empty joint fossa and may reveal the condyle anterior to the joint. Various treatment modalities have been described in literature for managing TMJ dislocation. This article reviews treatment modalities that have been used for treating TMJ dislocations along with a case report.

\section{Case report}

A 46-year-old female presented to the dental clinic complaining of inability to close her mouth and difficulty in talking for the past 4 months (Fig 1). Patient gave history of uneventful extraction of teeth 4 month prior to the present visit. No history of trauma or recent hospitalization was given. Patient was suffering from psychiatric illness and was not on any medication.
General physical examination revealed that the patient had an elongated face and was not able to close her mouth. Her speech was not clear. There was no visible evidence of contusion or surgical scar.

On palpation, TMJ and masticatory muscles were non tender, bilateral depression was found in the preauricular region with the condyle being palpated $3-4 \mathrm{~cm}$ in front of the depression on either side.

Intraoral examination revealed completely edentulous upper and lower jaws. Orthopantomogram revealed the right and left Condylar heads being positioned anterior to the glenoid fossa (Fig 2). A clinical diagnosed of bilateral chronic Condylar dislocation of TMJ was given.

Following infiltration of local anaesthesia in the pterygoid region, condyles were reduced manually. After reduction the joint was immobilized with the Barton's bandage. The patient was advised not to open the mouth wide and not to remove the bandage for a week. Patient was recalled after a week for the removal

Correspondence

Dr. Shrijana Shakya

Department of Oral medicine and Radiology

Manipal College of Dental Sciences, Mangalore

E-mail: shriju79@hotmail.com 
of the bandage. The patient was rehabilitated with the complete denture after 3 weeks. Patient was reviewed after 3 months without any recurrent dislocations
(Fig 3). Orthopantomogram was taken which showed the condyle in its normal position in the glenoid fossa (Fig 4).

Table 1: Treatment modalities for dislocation of TMJ

\begin{tabular}{|c|c|}
\hline Acute dislocation & Recurrent dislocation \\
\hline $\begin{array}{l}\text { Manual reduction } \\
\text {-Without local anesthesia } \\
\text {-With local anesthesia } \\
\text {-With muscle relaxant }\end{array}$ & $\begin{array}{l}\text { Manual reduction } \\
\text {-Followed by injection of sclerosing agents into joint } \\
\text {-Followed by injection of Botulinum toxin }\end{array}$ \\
\hline Induction of gag reflex & $\begin{array}{l}\text { Indirect reduction } \\
\text {-Via sigmoid notch } \\
\text {-Preauricular approach }\end{array}$ \\
\hline & $\begin{array}{l}\text { Direct reduction } \\
\text {-Restitution of capsular ligament } \\
\text {-Augmentation of articular eminence } \\
\text {-Eminectomy } \\
\text {-Condylotomy } \\
\text {-Myotomy }\end{array}$ \\
\hline
\end{tabular}

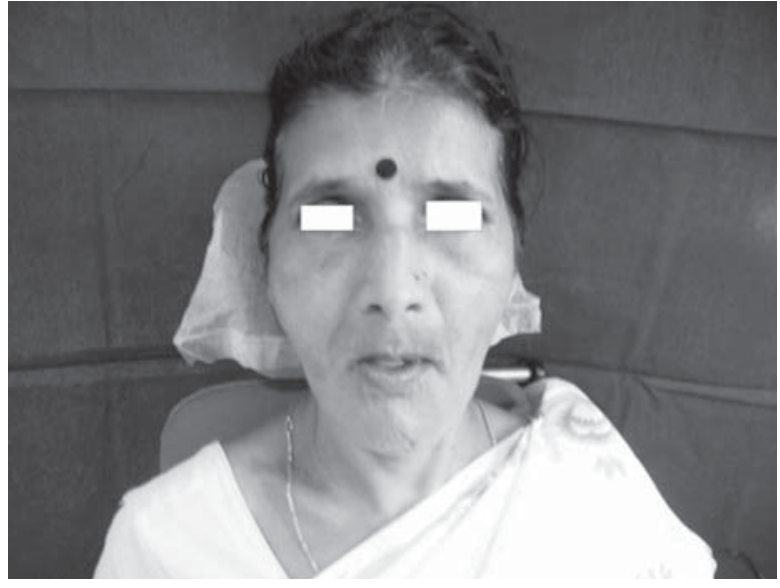

Fig 1: Clinical Photograph shows elongated face

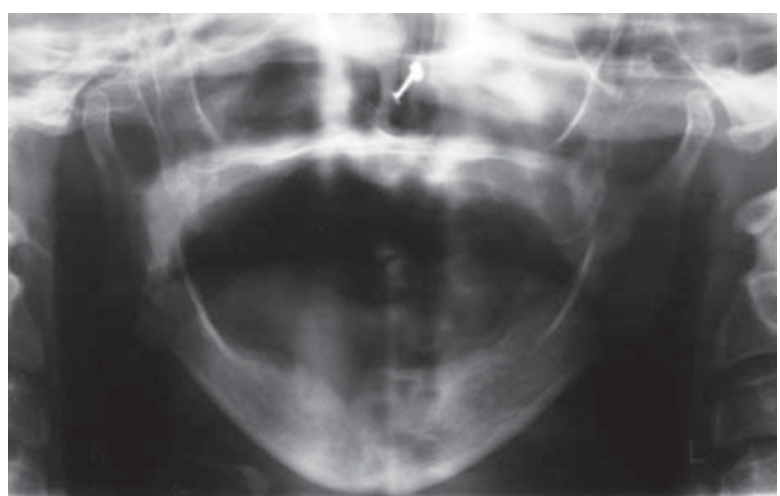

Fig 2: Orthopantomogram shows bilateral dislocation of the mandibular condyles

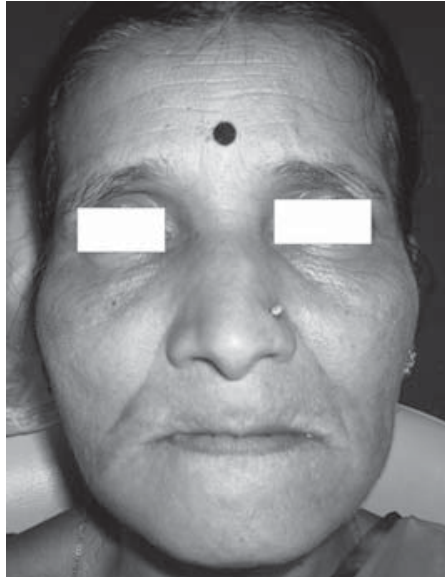

Fig 3: Three month follow up clinical photograph

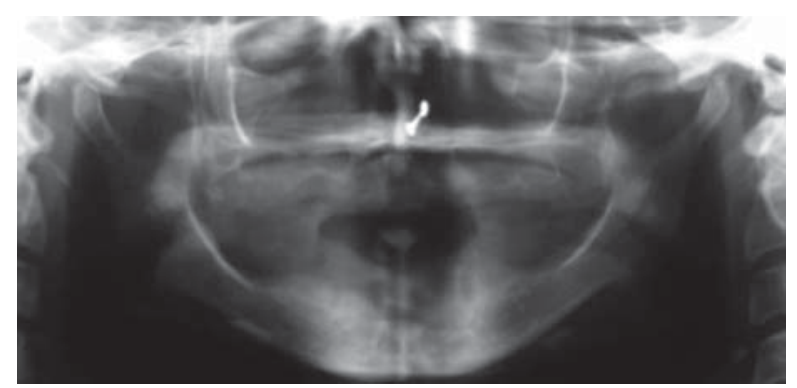

Fig 4: Orthopantomogram taken during the 3 month follow up visit shows normal position of the mandibular condyles 


\section{Discussion}

In 1832, Sir Astley Cooper proposed the principles for diagnosis and treatment of dislocation and used the term complete dislocations and imperfect dislocation for luxation and subluxation respectively ${ }^{3}$. TMJ luxation differs from subluxation in that in the later case the condyle moves anterior to the articular eminence during mouth opening but reduces to its normal position without manipulation ${ }^{4}$. Dislocation of TMJ occurs in up to $7 \%$ of people during their lifetime ${ }^{5}$. TMJ dislocation represents $3 \%$ of all articular body luxation ${ }^{6}$. When this condition progresses and becomes frequent it is termed as recurrent dislocation or habitual dislocation. The problem can affect any age group from under the age of 10 years up to 79 years but more common in $2^{\text {nd }}$ and $3^{\text {rd }}$ decade $^{7}$. Highest frequency of TMJ luxation is found in females, the cause for this is still unknown ${ }^{3}$.

The condition is characterised by the inability to close the mouth with protrusion of the mentalis, spasm and tension in the masticatory muscle, excessive salivation and difficulty in speech ${ }^{6}$ Depression may be noted in preauricular area normally occupied by condyles. The inability to close the mouth is due to the spasm of the masticatory muscles. A typical facial expression is due to anxiety related to the thought of not being able to close the mouth.

It occurs as a combination of 3 factors; Laxity of mandibular and capsular ligaments, large bony eminence and spasm of muscle. It may also be due to excessive and irregular movement of the disc condyle complex ahead of articular eminence or due to spasm of temporalis muscle initiated by myotactic reflex ${ }^{3}$.

Various factors may lead to dislocation like habitual wide opening of mouth, when yawning ${ }^{8}$ or eating, procedures that necessitate prolonged mouth opening procedure like in third molar extraction, endodontic treatment, endotracheal intubation, laryngoscopy ${ }^{9}$, transoral fibrotic bronchoscopy ${ }^{10}$, steep articular eminence found in skeletal deep bite facial type. Certain medications used for Psychiatric problem may also lead to dislocation ${ }^{11}$. Some people may be genetically predisposed to joint laxity like in cases of Ehler Danlos syndrome ${ }^{12}$, Marfan syndrome. Whatever the cause, instable joint is very likely to undergo dislocation. The factor that determines the instability of the joint may be due to integrity of the ligament, activity of the musculature acting on the joint and alteration in the bony architecture of the joint ${ }^{3}$.

\section{Classification}

Depending upon the position the condyle occupies ${ }^{13}$ Heslop in 1956, described the anterior dislocation in which the condyle moves anterior to the articulating eminence. It is one of the most common type of dislocation It represents the pathological forward extension of normal translatory movement of head of condyle. The antero-lateral variant was described by Morris and Hutton in 1957.

Helmy in 1957 described the posterior variant in which the head of condyle is displaced posterior to its usual position. It is usually associated with a fracture of base of skull or the anterior wall of bony meatus.

Lateral dislocation has been described by Allen and young in 1969 in 2 subgroups. Type 1 is the lateral subluxation and type 2 is a complete dislocation where the condyle if forced laterally and superiorly to the temporal fossa. It is accompanied by the fracture of body of mandible at symphysis.

Superior dislocation as described by Zecha in 1977 is the dislocation of condyle in the middle cranial fossa and associated with fracture of glenoid fossa. It is said to be most probably due to the small rounded shape of the condyle which fails to impinge in the margins which is stronger than the central area.

\section{Management}

Many treatment modalities are aimed at pain management and minimize dysfunction associated with of recurrent TMJ luxation (Table 1). Conservative methods promote some temporary relief of symptoms and recurrence is common while surgical interventions are normally more effective for definite treatment. Comparisons of the various types of modalities are difficult due to variation in postoperative follow up and different definitions of success rate.

The most conservative method for treatment is the manual reduction of the joint as described by Lewis $1981^{14}$. It is achieved by making the patient sit down and the clinician standing in front or at 11'0 clock position. Then pressing the thumb down on the occlusal surface of lower molar teeth. At the same time elevating the chin with the fingers and pushing the entire mandible posteriorly. Fordyces (1965) emphasised the downward pulling of the chin before pushing it backwards as in original Hippocratic method.

A modification of this method is achieved by placing the thumb intraorally on external oblique ridge in the mandibular molar region and the finger extraorally under the lower border of mandible the thumb is protected in this method from accidental biting of the patient while reducing the dislocation ${ }^{15}$.

Sometime this procedure may be difficult because of the reflex spasm of the muscles, in these cases local 
anaesthesia can be used. It is based on the theory that dislocation is maintained by muscle spasm and secondary to painful stimuli arising from capsule. In this procedure Lignocaine hydrochloride is injected in the joint and following which reduction occurs spontaneously or in some cases manual reduction as said above will be required. Even unilateral injection may be adequate in case of bilateral dislocation due to elimination of neuromuscular reflex which extends over the muscle. In the apprehensive and anxious patient local anaesthesia may be assisted by sedation using intravenous diazepam.

Another method of reduction was described in 1987 by Awang. He advised a simple, safe and rapid procedure of reduction in case of acute dislocation by activating the reflex neuromuscular activity. According to him, induction of a gag reflex by probing the soft palate resulted in reduction ${ }^{16}$.

The use of various sclerosing agent ${ }^{3}$, like alcohol, rivanol (aethacridine), 5\%sodium psylliate (sylnasol), sodium morrhuate, $3 \%$ sodium tetradecyl sulfate have been injected in to the joint cavity. The procedure involves injection of the chemicals in the upper joint cavity guided by arthroscopy. Rationale for injecting these agents is to cause fibrosis and limit the jaw movement. Immobilization of the joint was then achieved by intermaxillary fixation for a period of 3-6 weeks. This method is not used routinely because the extent of fibrosis cannot be controlled.

Use of autologous blood injection into the joint to prevent frequent dislocations has been reported as a non surgical method for frequent dislocation in older individual. Blood injected into the upper joint space and around the joint would lead to scarring and fibrosis restricting the mandibular movements. The use of this technique is still debatable in case of younger patient and in those having articular degenerative diseases and rheumatoid arthritis ${ }^{17,18}$.

Another method used for joint dislocation is the introduction of botulinum toxin in to the joint. Botulinum toxin is derived from the anaerobic, Gram positive rod clostridium botulinum. Serologically it can be differentiated into 8 types. Among these, typeA botulinum was used for treatment of recurrent dislocations as a result of impaired muscle coordination secondary to oromandibular dystonia, neuroleptically induced early and brain stem syndromes of various origins. It is a relatively conservative procedure with the reversible effect requiring repeated injections every 2-4 months. The treatment can be given on an outpatient basis. The adverse effect of treatment involves diffusion into adjacent tissues, transient dysphagia, nasal speech, painful chewing, nasal regurgitation and dysarthria. This treatment is contraindicated in disease affecting neuromuscular transmission like myasthenia gravis (Lambert Eaton Syndrome) and in pregnant and lactating women ${ }^{19,20}$.

There are many different surgical approaches for the treatment of dislocation. In 1968, Rowe and Killey used a bone hook which was passed over the Sigmoid Notch through a small incision below the angle of mandible and downward traction was applied on condyle.

In 1976, Adekeya et al described a technique in which the occlusion was restored by means of inverted $\mathrm{L}$ shaped osteotomy of ramus. Other type of osteotomy like vertical and horizontal were not used because of the possibility of interference from coronoid process.

In 1981, Lewis used a technique in which the Bristows elevator was passed through the temporal fascia as in Gillies technique of elevation of depressed zygomatic bone. The tip of the elevator was used and strong force was applied in downward and posterior direction. This method also facilitates open reduction by extending the incision as used for preauricular approach to the joint ${ }^{14}$.

Open surgical methods includes various graft procedures like restitution of capsule and ligament. It can be achieved by exposing the lateral aspect of the TMJ and temporal fascia for a distance of about $2.5 \mathrm{~cm}$ above the glenoid fossa. The strip of the capsule is excised from the outer surface and the margins are sutured together. Suture line is reinforced by turning down the flap of temporal fascia and securing it to capsule and ligament. Immobilization is not required because the edema that is produced will limit the mandibular movement. Limitation of forward movement can be done by tying a length of fascia lata or Mersilene (Dacron ) to zygomatic arch and around Condylar neck ${ }^{3}$.

Augmentation of the articular eminence can be done by Iliac or calvaria bone graft ${ }^{21}$. The space needed for graft is created by reflecting the periosteum of the zygomatic arch and articular eminence and displacing it posteriorly. The outcome of these types of surgeries varies in terms of resorption. Iliac bone graft was seen to have resorption whereas in case of the calvarial graft complication was seen in the donor site with the occurrence of dural tears, arachnoidal bleeding hematoma formation and scalp infections. Since the iliac graft showed remarkable degree of resorption bovine cartilages were tried. Cartilage were found to be dimensionally stable in terms of resorption which was further enhanced by their calcification. 
Various other materials have also been used like Lshaped pins, vitallium mesh implants, and mini implants to enhance the eminence ${ }^{22,23}$. Uses of these materials have been reported with increase incidence of fracture and loosening under functional loads. Silicon blocks used in the shape of wedge has been reported to evoke immune reaction and also showed displacement as a result of functional movement. Use of porous coralline hydroxyapatite ${ }^{24}$ into the joint space has also been reported with displacement under function. Among all different treatment modalities studied in the literature, a rate of $95 \%$ of cases without recurrence both after eminectomy and use of metallic implant over the articular eminence ${ }^{3}$.

Myrhaug in 1951 firstreported eminectomy as a treatment for dislocation. Removal of eminence will facilitate the return of the condyle without any interference into the glenoid fossa. Although dislocation takes place, painless reduction will occurs automatically. Both techniques eminectomy and use of bone miniplates in the articular eminence for the treatment of recurrent temporomandibular joint were found to be effective ${ }^{4}$.

Augmentation of the articular eminence by displacement of the zygomatic arch and obstructing the path of the Condylar translation has been first described by Mayer in 1933 since then it has been modified by various authors. Dautrey ${ }^{25}$ modified the technique in which the green stick fracture was performed at zygomaticotemporal suture, which provides the rebound elasticity and provide stability in the altered position. It cannot be performed in elder people due to the brittleness of the bone; so is restricted to the younger individual.

Various other methods like Condylotomy ${ }^{26}$, modified condylotomy $^{27}$ and myotomy had been tried. Condylotomy was used as a blind approach using a modified Kostecka approach and Gigli saw. It is an open approach usually using the pre auricular route. Myotomy $^{28}$ with resection of insertion of external pterygoid muscle had been described by Bowman in 1949. It limits the mandibular translation and allows only rotational movement of condyle. This is followed by intermaxillary fixation for about 10 days. Some drawbacks of this method are reunion of the muscle during healing, risk of bleeding in the area and impaired vision to the site.

\section{Conclusion}

Even though, various surgical and other invasive methods have been tried for recurrent and chronic cases, all cases irrespective of the duration of occurrence should be initially reduced manually when the muscles are in the state of relaxation. This insures the quick painless reduction of the condyle.

\section{References}

1. Casone P, Ungari C, Paparo F, Marianetti TM, Ramieri V, Falone M. A new surgical approach for the treatment of chronic recurrent temporomandibular joint dislocation. J Craniofac Surg. 2008;19(2):510-2.

2. Magge SN, Chen HI, Heuer GG, Carrasco LR, Storm PB. Dislocation of mandible into the middle cranial fossa -a case report. J Neurosurg. 2007;107(1):75- 8 .

3. Shorey CW, Campbell JH. Dislocation of the temporomandibular joint. Oral Surg Oral Med Oral Pathol Oral Radiol Endod. 2000;89(6):6628.

4. Cardoso AB, Vanconcelos BC, Oliveira DM. Comparative study of eminectomy and use of bone miniplate in articular eminence for treatment of recurrent temporomandibular joint dislocation. Rev Bras otorrinolaringol. 2005;71(1):32-7.

5. Tesfaya Y, Skorzewska A, LAl S. Hazard of yawning. CMAJ. 1991;145(12):1560.

6. Lovely FW, Copeland RA. Reduction eminoplasty for chronic recurrent luxation of the temporomandibular joint. J Can Dent Assoc. 1981; 47(3):179-84.

7. Tasanen A, Lamberg MA, Closed condylotomy in treatment of recurrent dislocation of mandibular condyle. Int $\mathrm{J}$ Oral Surgery. 1978;7(1):1-6.

8. Avidan A. Dislocation of the temporomandibular joint due to forceful yawning during induction with propofol. J Clin Anesth. 2002;14(2):15960.

9. Rattan V,AroraS. Prolonged temporomandibular joint dislocation in an unconscious patient after airway manipulation. Anesth Analg. 2006;102(4):1294.

10. Durga VK, Millns JP, Smith JE, Manoeuvers used to clean the airway during fibreoptic intubation. Br J Anaesth. 2001;87(2):207-11.

11. Undt G, Weichselbraun A, Wagner A, Kermer C, Rasse M. Recurrent mandibular dislocation under neuroleptic drug therapy, treated by bilateral eminectomy. J Craniomaxillofac Surg. 1996;24(3):184-8.

12. Poveda-Roda R, Bagán JV, Díaz-Fernández JM, Hernández-Bazán S, Jiménez-Soriano Y. Part I Review of temporomandibular joint pathology. Part I: Classification, epidemiology and risk factors. Med Oral Patol Oral Cir Bucal. 2007;12(4):E292-8

13. Bradley P, James D, Norman JE de B. Injuries of condyle and coronoid process: In: Williams 
J L, editors. Rowe and Williams Maxillofacial injuries. UK: Churchill Livingstone. P. 405-73.

14. Lewis JE. A simple technique for reduction of long standing dislocation of mandible. Br J Oral Surg. 1981;19(1):52-6.

15. Howe GL Minor oral surgery 3rd ed. Wright: Bristol; 1985. P. 393-5.

16. Awang MN. A new approach to the reduction of acute dislocation of the temporomandibular joint: a report of three cases. Br J Oral Maxillofac Surg. 1987;25(3):244-9.

17. Hasson O,NahlieliO.Autologousbloodinjection for treatment of recurrent temporomandibular joint dislocation. Oral Surg Oral Med Oral Pathol Oral Radiol Endod. 2001;92(4):390-3.

18. Kato T, Shimoyama T, Nasu D, Kaneko T, Horie N, Kubo I. Autologous blood injection into articular cavity for the treatment of recurrent temporomandibular dislocation: a case report. J Oral Sci. 2007;49(3):237-9.

19. Daelen B, Thorwirth V, Koch A: Treatment of recurrent dislocation of the temporomandibular joint with type A botulinum toxin. Int J Oral Maxillofac Surg. 1997;26(6):458-60.

20. Ziegler CM, Haag C, Muhling J. Treatment of recurrent temporomandibular joint dislocation with intramuscular botulinum toxin injection. Clin Oral Investig. 2003;7(1):52-5.

21. Medra AM, Mahrous AM. Glenotemporal osteotomy and bone grafting in the management of chronic recurrent dislocation and hypermobility of temporomandibular joint. Br J Oral Maxillofac Surg. 2008;46(2):119-22.
22. Shibata T, Yamashita T, Nakajima N, Ueda M, Ishijima T, Shigezumi M, et al. Treatment of habitual temporomandibular dislocation with miniplate eminoplasty: a report of nine cases. J Oral Rehabil. 2002;29(9):890-4.

23. Kuttenberger JJ, Hardt N. Long term results following miniplate eminoplasty for treatment of recurrent dislocation and habitual luxation of temporomandibular joint. Int J Oral Maxillofac Surg. 2003;32(5):474-9.

24. Karabouta I. Increasing the articular eminence by the use of blocks of porous coralline hydroxyapatite for treatment of recurrent TMJ dislocation. J Craniomaxillofac Surg. 1990;18(3):107-9.

25. Lawlor MG. Recurrent dislocation of the mandible: treatment of ten cases by the Dautery procedure. Br J Oral Surg. 1982;20(1):14-21.

26. Albury CD. Modified condylotomy for chronic non reducing disk dislocation. Oral surg Oral Med Oral Pathol Oral Radiol Endod. 1997;84(3):234-40.

27. Hall HD, Navarro EZ, Gibbs SJ. Prospective study of modified condylotomy for treatment of non reducing disk displacement Oral surg Oral Med Oral Pathol Oral Radiol Endod. 2000;89(2):147-58

28. SindetPS. Intraoral myotomy of lateral pterygoid muscle for treatment of recurrent dislocation of mandibular condyle. J Oral Maxillofac Surg. 1988;46(6):445-9. 